\title{
Simulation of power quality in residential electricity networks
}

\author{
D. McQueen ${ }^{1}$, M. McQueen ${ }^{2}$, P. Hyland ${ }^{3}$, S. Watson ${ }^{1}$ \\ ${ }^{1}$ Centre for Renewable Energy Systems Technology \\ Loughborough University \\ Leicestershire LE11 3TU, United Kingdom \\ phone: +44 1509 223466, fax: +44 1509 610031, email: D.Mcqueen@lboro.ac.uk, S.J.Watson@lboro.ac.uk \\ ${ }^{2}$ School of Information Technology and Electrotechnology \\ Otago Polytechnic \\ Dunedin, New Zealand \\ phone: +64 3 4796196, fax: +64 3 4776032, email: MalcolmM@tekotago.ac.nz \\ ${ }^{3}$ AuStral Engineering Associates Limited \\ Dunedin, New Zealand \\ phone: +64 3 4792555, fax: +64 3 4792888, email: P.Hyland@aeal.co.nz
}

\begin{abstract}
.
The prevalent engineering practice (PEP) for demand estimation in Low Voltage electricity networks is based on an After Diversity Maximum Demand (ADMD) modified by a Diversity Factor. This method predicts the maximum likely voltage drop, accounting for diversity. However, it is inconsistent with both the nature of demand, which can be represented as a stochastic process, and with the wording of power quality standards such as BS EN 50160. This paper develops a numerical method that is then applied to a case study.
\end{abstract}

A programme is undertaken to meter residential electricity demand. Using this data a generalised mathematical model is developed. The model is applied in a Monte Carlo simulation of the three-phase Low Voltage network. A house that was the subject of a power quality complaint, which resulted in network reparation, is chosen for the case study. A retrospective analysis is performed to hindcast the power quality received before the reparation, using both the Monte Carlo method and PEP; this is compared with a voltage trace recorded at the time of the complaint. The analysis is then used to model the power quality for the present network design and compared with measurements. The model is also used to examine phase unbalance, and the effect of sampling interval on measured demand.

\section{Keywords}

Low Voltage, Distribution networks, Monte Carlo, Voltage Drop, Voltage imbalance

\section{Introduction}

The Prevalent Engineering Practice (PEP) used by the electricity industry for sizing conductors in Low Voltage (LV) distribution networks can be traced to J Boggis in the 1950's [1]. The methodology is based upon estimating the maximum demand that is likely to be placed by a group of customers inside of a time window that is commonly taken to be a year. From this the greatest likely voltage drop to occur in normal operating conditions can be calculated.

When analysis of electricity demand is made from a risk based perspective where statistical coherence is necessary this method is found wanting. Further, newer design standards that have been written that require a greater understanding of the nature of the demand and the estimation of the time duration curve for received voltages.

Electricity demand placed by individual consumers is a fluctuating function of time. The demand (household load profile) is made up by operation of appliances that are activated either by consumers or by control systems such as thermostats; both of these are stochastic processes. As such, appliance actuations are largely independent, as are the loading patterns placed by neighbouring houses. There are some overall driving factors that will increase the tendency for appliances to be used coincidently, such as the time of day and the ambient temperature. However, the result is that residential electricity demand is largely a stochastic process and therefore exhibits diversity.

Infrastructural networks benefit from diversity since the maximum demand placed by a group of customers is less than the sum of the maximum demands of the individuals; in other words peak demands are very unlikely to be co-incident. Network designers use this to their advantage by being able to reduce capital investment. This is embedded within PEP.

In this paper we seek to develop and demonstrate a better method for estimating residential electricity demand. This method uses the assumption that the demand is a stochastic process, and a Monte Carlo simulation is used to calculate the expected voltage profile. The Monte Carlo method is shown to be flexible and give added benefits through the prediction of conductor losses, and 
phase unbalance. An analysis is also undertaken to demonstrate the effect that sampling interval has on the aggregate demand placed by a group of customers.

\section{Low Voltage Network Design}

\section{A. Power Quality}

In a typical electricity infrastructure the LV network's purpose is to provide connection from the terminal end of the Medium Voltage (MV) network to the points of supply for individual customers. Typically a discrete LV network will consist of a transformer to step down the voltage from the MV network to a distribution voltage of $400 \mathrm{~V}$ phase to phase. Beyond the distribution transformer the network is likely to be a tree (although rings are not uncommon) and will consist of three phase conductors, a return conductor, and protection devices. Voltage regulation is likely to be passive.

There are many elements to the design of the LV network, such as determining the necessary tensile strength for overhead conductors, the height of poles to ensure conductors do not sag below specified heights etc. In this paper we are solely concerned with the design aspect that ensures received voltages stay within regulation limits.

It is helpful to identify the types of adverse power quality symptom that the design methods are attempting to counter. The focus here is upon voltage drop events; voltage drops to between 0.1 and $0.9 \mathrm{pu}$ (per unit, where a unit is the nominal voltage) that have duration greater than 1 minute [2]. These are largely the result of the overloading of conductors during either anomalous loading or the result of undersized conductors. It is the second cause that is of concern to the LV network designer.

\section{B. Prevalent Engineering Practice}

The electricity industry has for a long time taken advantage of diversity. A standardised formulation to calculate the maximum load from a group of customers was developed by Boggis in 1953 [1]. The formulae, which form PEP, are based upon the concept of an After Diversity Maximum Demand (ADMD). The ADMD is the maximum demand, per customer, as the number of customers connected to the network approaches infinity. This is usually derived from the maximum yearly nodal demand on the transmission network divided by the number of customers served by that network. Many utilities will use further analyses to attribute ADMDs to different customer classes (e.g. residential, commercial, industrial, or even finer grained). The ADMD is multiplied by a diversity factor (DF), this increases the demand per customer as the size of the group decreases. The PEP formulae can take many forms but generally resembles Equation 1, the ADMD defined by Equation 2, and the diversity factor (DF) given by Equation 3.

$$
M D=N \times D F \times A D M D
$$

$$
A D M D \quad=\lim _{n \rightarrow \infty} \frac{1}{n} \sum_{n=1}^{n} I_{n}
$$

$$
\begin{aligned}
& D F=1+\frac{k}{N} \\
& \text { Where } M D=\text { maximum demand } \\
& D F=\text { diversity factor } \\
& A D M D=\text { After Diversity Maximum Demand } \\
& I=\text { current per customer at time of system } \\
& \text { maximum demand } \\
& N \quad=\text { number of customers } \\
& k \quad=\text { coincidence factor. }
\end{aligned}
$$

The greatest failing of PEP is that it calculates the likely maximum demand for a group of customers within a specific period of time, usually one year. And, demand monitoring typically uses an industry standard 30-minute sampling rate. This translates to a statistical confidence level of $99.994 \%$, which from a risk analysis point of view is an extremely cautious approach to design. Further PEP gives no further information, such as the time of system peak or the demand at times other than the network peak.

The time window that network design methods use is important to define. Loads will change with time due to changes in appliance stocks, user behaviour patterns, and geographical changes (emigration/ immigration from an area, building of new houses etc.) [3]. It is likely that many electricity networks have benefited from conservative design practices that have unintentionally accounted for load growth. An example time window used for PEP is a one-year period where the load is forecast for ten-years time. In developing the methodologies in this paper load forecasts are for the present year, this could be manipulated to achieve the objectives of an economically optimised risk analysis. Other points to consider when modelling demands are the effects of load control and embedded generation, these are not considered here.

\section{Electricity Regulations}

The New Zealand electricity regulations are written in a manner consistent with PEP. Stating that "received voltage must be within $\pm 6 \%$ of 230 V apart from momentary fluctuations" [4]. The term "momentary fluctuations" is used to cover fault and irregular loading conditions. More recent standards such as EN 50160 have been written with a statistically coherent emphasis; stating that "during each period of one week $95 \%$ of the 10-minute rms values of the supply voltage shall be within $U_{n} \pm 10$.", where $\mathrm{U}_{\mathrm{n}}$ is the nominal voltage [5]. Effectively this allows for the inevitable chance that voltage will diverge from the regulation bounds.

Departures of voltage out of the regulation bounds will not necessarily be met by voltage quality complaints. The 
following conditions must be satisfied before the threshold for voltage quality complaints is passed:

- The customer has to be aware of the symptoms of voltage drops.

- The voltage drop must be of a magnitude that causes appliances to malfunction or lights to dim.

- The voltage drop must occur at a time when the customer is using electricity.

- The voltage drop must occur enough times to become an irritant.

Further factors may increase the likelihood that a complaint will be made such as rapid changes in voltage or repeated voltage drops. On the whole however it is unlikely that a complaint will be made unless there is a significant departure from regulation bounds [6]. These factors further complicate the problems of determining what are acceptable design standards and hence the appropriate methods for reaching those designs.

A study of the New Zealand electricity industry revealed that the majority of distribution companies use PEP for low voltage network design [7]. The study also revealed that the number of voltage quality complaints attributable to network design is extremely small. Thus designs are not necessarily reaching their economic optimal. Hence, PEP is not the best approach for obtaining an optimised solution for network design.

\section{Demand Modelling}

Solution of voltage drop problems within a statistically consistent paradigm has been made using an analytical solution [8] by addition of Beta functions. However, these solutions become awkward when considering branched networks with a large number of customers. Another common method for demand estimation is "bottom up modelling", whereby the demand for every appliance is simulated [9]. Models of these types requires knowledge of appliance demand patterns and are very useful in trying to assess demand side management schemes, however prove information intensive when trying to replicate generalised household load profiles.

The methodology developed here assumes that electricity demand is a stochastic process and constructs representative loading profiles for each consumer. The loading profiles are then injected into a model representative of the network impedance; hence the voltage drop can be solved in a Monte Carlo simulation. This allows the voltage and conductor current loading profiles to be determined and hence the distribution of voltages received at each premises, giving a statistically informative description of power quality.

The assumption is made that all houses have the same probability of producing any individual loading profile. This is important since it is not known what the energy demand of customers will be in the design stage or even within an existing network. Where anomalous loads are causing fault conditions it is possible to inject an additional load into the network model.

\section{A Mathematical Model for Residential Electricity Demand}

To support the Monte Carlo methodology a mathematical model must be built for residential electricity demand. The objective of developing a mathematical model is so that representative load profiles can be formed for "any" customer. Hence the modelling procedure must generalise upon the sample gathered in a monitoring programme. The model must also parameterise the data in such a way that it can be manipulated to best represent the population being modelled, use a methodology that can be easily replicated and deployed within applications. And lastly, it is preferable that the model be intuitive.

A scoping study for the methodology based upon a data set from customers in the Auckland region (New Zealand) highlighted several areas for further investigation such as determining the appropriate sampling interval to use [7]. A further metering program was instigated and results from this study are used to develop the models used here. The program measured electricity demand for 21 houses at a 1-minute sampling interval for a period of approximately 1 -week per house. (See Section 4 for details of the effect of a change in sampling interval. For the main part of this analysis a sampling interval of $1 / 2$ an hour has been used, as this is a standard sampling rate used in the electricity industry).

The mathematical modelling process can be segregated into two procedures. Firstly, the measured data are parametrised in such a way that the objectives of modelling are met. This basically entails isolating those dynamics that are responsible for diversity which are assumed universal, from those that are specific to the population being modelled.

The second part of the procedure is to generate simulated load profiles using the information obtained during the parametrisation. This involves random number generation and building the load profile.

In the research conducted by D. McQueen many types of models were investigated including the use of Markov chains, Fourier series and Poisson Rectangular Pulses [10]. However, the model used for this analysis is based upon a simple representation for the demand within each time section based upon a Gamma distribution.

The mathematical model here is formed in the following way (from the metered data):

- The daily energy use (scale parameter) for each load profile is found (Equation 4). The distribution of daily energy use over all houses and all days is well represented using a Gamma distribution.

- The scaled daily load profile for each day is found (such that the integral of each load profile is one). Scaled load profiles from all houses are binned together (Equation 5).

- A Gamma distribution is fitted to the load in each time section for the binned group.

Then for each simulated load profile: 
- A random daily energy use is generated.

- For each time section a random demand is generated such that a normalised daily load profile is formed.

- The normalised load profile is multiplied by the energy use such that a simulated load profile is formed.

$$
S_{d, h}=\sum_{t=1}^{24} P_{d, h}(t) \begin{cases}t=1: 0.5: 24 \\ d=1,2, \ldots, & D \\ h=1,2, \ldots, & H\end{cases}
$$

where

$$
\begin{array}{ll}
P & =\text { a load profile } \\
S & =\text { scale parameter } \\
d & =\text { day index } \\
h & =\text { house index } \\
t & =\text { time } \\
N_{d, h}(t)=\frac{P_{d, h}(t)}{\sum_{t=1}^{T} P_{d, h}(t)}
\end{array}
$$

where $N=$ scaled load profile

Temperature dependence is added to the demand model, such that the mean daily energy demand becomes a function of temperature.

During the monitoring study a distribution transformer was also monitored so that the hypothesis "individual household loads are independent" could be tested. The transformer loading profiles were also used to test the ability of the mathematical demand models to simulate the network loading conditions. Figure 1 gives cumulative density functions (CDF) for the loading measured on each of the three phases and for the transformers' total loading during the monitoring period. Beside each CDF is the corresponding function generated using the mathematical model simulating loading for the monitoring period.

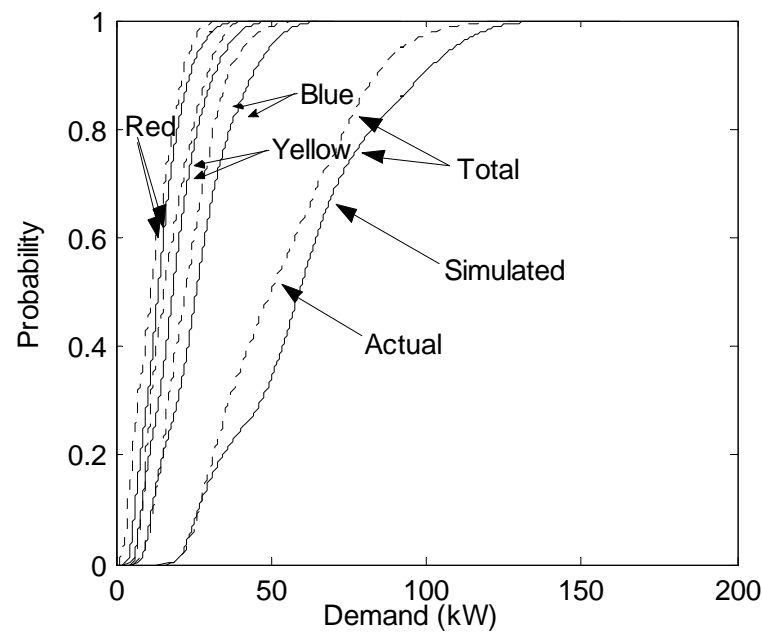

Fig. 1, Cumulative density functions for the demand on the distribution transformer (solid line) along side the matching functions formed from the simulated demands (dotted lines).
CDFs are given for phases as well as the total transformer demand.

The analysis of distribution transformer loading has been expanded to cover modelling of the loss of insulation life for a network located in New Zealand [11]. The analysis couples models for ambient temperature profiles and customer demand to recreate the thermal performance of the transformer and hence the insulation "hot spot". Loading predictions from the model are shown to agree very well with measurements taken by Maximum Demand Indicators (MDI), giving greater confidence in the methodology used here.

\section{Voltage Quality Case Study}

To demonstrate the Monte Carlo methodology a case study is presented. A particular house has been identified because it was the focus of a voltage quality complaint and subsequent network reparation. The complaint was lodged because of noticeably low voltage affecting appliances, in particular a facsimile and a sun-bed. Voltage traces taken at the time revealed voltages as low as $85 \%$ of nominal. Calculations made at the time using PEP predicted a voltage received at time of maximum demand of $87 \%$ of nominal (ADMD $4 \mathrm{~kW}$, Diversity factor 3). The result being that much of the network was redesigned; many of the conductors were upgraded and several houses put onto different phases (see Appendix A for details of conductor types).

A street survey was used to determine which phases customers are connected and exact conductor section lengths. This coupled with information drawn from the Network companies' impedance manual [12] is used to determine the network impedance characteristics. Figure 2 is an aerial photograph of the network, with phases marked for each house.

The representation of the electrical network included the following assumptions:

- Single neutral current return path; the network is actually a multiply earthed neutral network.

- Constant conductor impedance (no thermal model used).

- Constant conductor separations (in accordance with the Network companies' impedance manual [12]).

- Loads are constant current sources.

- Transformer sending voltage is constant; equal to nominal.

- The voltage drop between the street (point of supply) and the power point, where voltage is measured, is not included. 


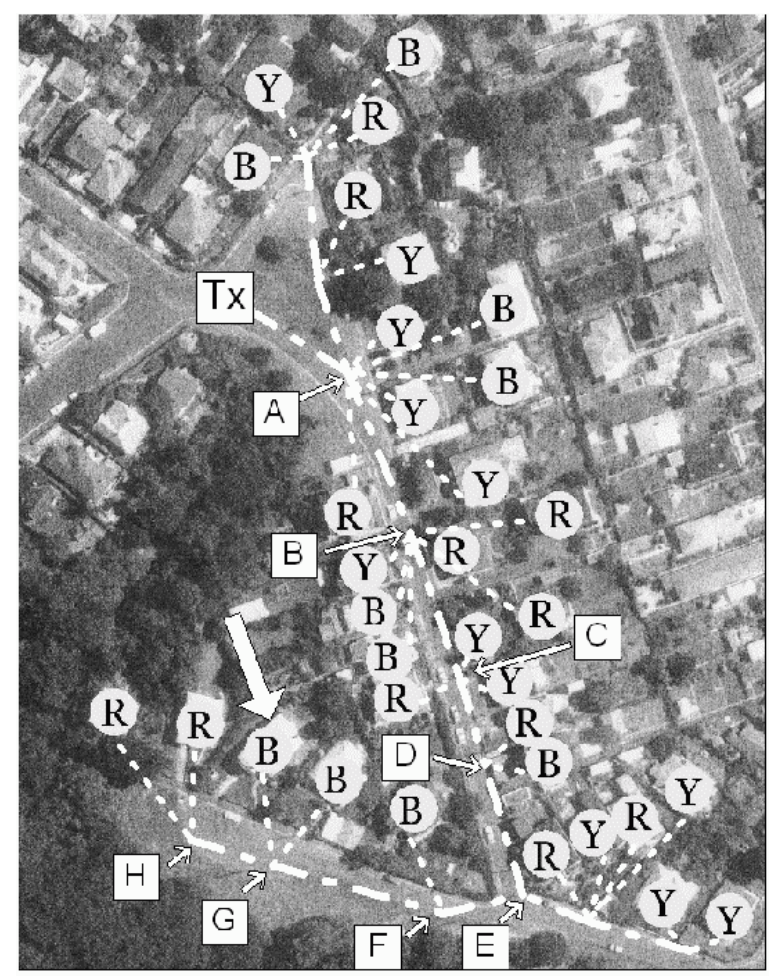

Fig. 2. Aerial photo showing Stonelaw Tce network's present design. The transformer is marked "Tx", houses are marked by circles with their phase marked R (red), Y (yellow, B (blue). The squares point to the ends of conductor sections used in the analysis.

Many of these assumptions can be improved upon however in this case study a simplistic approach is taken so that the methodology can be demonstrated. The assumptions also make it possible to solve the voltage drop calculation within each time section as a linear function without having to solve an iterative method such as the Newton-Rhapson. This will introduce errors; these have not been quantified within this research.

PEP is applied to calculate the lowest expected voltage at each node along the network (Figure 3). This has been performed for both the previous design and the present design. The house from which the voltage complaint originated is connected at the terminal of section $\mathrm{G}$. Calculations made using PEP agree with those made by design engineers at the time, considering that the $87 \%$ calculated is applicable to the terminal of section E. The repaired network shows that voltages on all phases are acceptable.

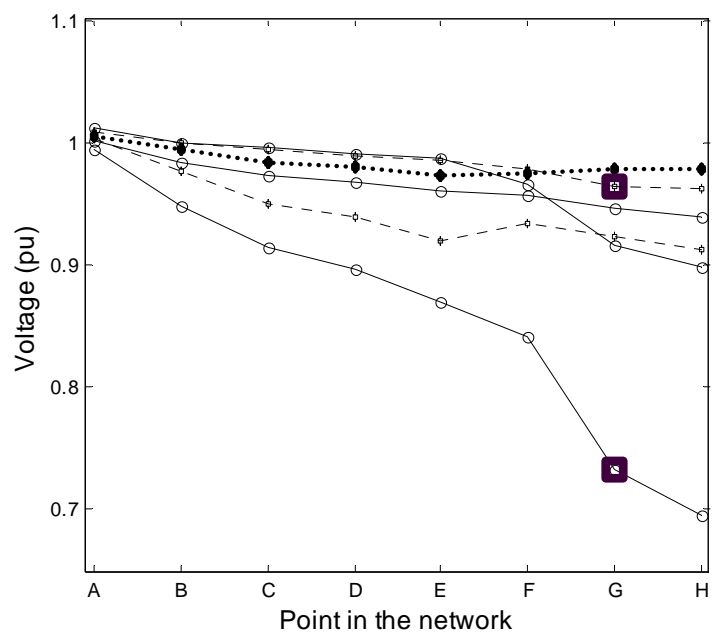

Fig. 3, Results of voltage drop calculations performed using PEP. Each line gives the total voltage drop for a phase. The solid lines give the previous design; the dotted lines give the present design. The appropriate position of the case study house is marked by the black square in both design cases. N.B. units are pu, that is "per unit" i.e. 1 unit is nominal voltage.

During the monitoring program the received voltage at the house was measured using a probe inserted into the power point closest the fuse board. The monitoring period for the house lasted two weeks.

A Monte Carlo simulation is performed to predict the voltage received at the house during the monitoring period. The following information is also used in this application:

- The temperature range is restricted to that measured during the monitoring programme.

- The Network companies' database is used to find the energy demand for customers in this network (records exist for about $90 \%$ of connected houses).

- Energy and demand patterns are considered to be the same before and after the network reparation event (the same assumption applies to the PEP analysis).

Results of the simulation over the monitoring period are presented in the form of CDFs formed for the measured data, the simulation for the present design, and the simulation for the previous design (see Figure 4). The measured data exhibit a greater range of voltage and a higher sending end voltage than the results of the simulation for the present design. However they are positive and show good agreement given the assumptions made about the electrical characteristics of the network. Results are also added to the figure for the voltage range recorded at the time of the complaint, by manually reading the analogue voltage trace. 


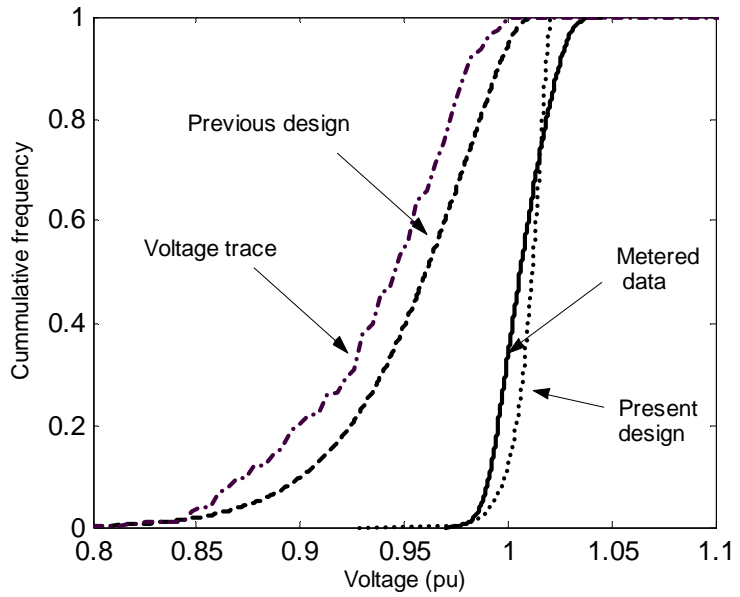

Fig. 4, Cumulative distribution functions for voltage received at premises of case study. The CDF formed from the metered data is given by the solid line. The CDF formed manually from the voltage trace recorded at the time of the complaint is given by the dash-dotted line. The previous (dashed) and present design (dotted) CDFs are formed from the Monte Carlo simulation for the monitoring period.

Next the Monte Carlo simulation is extended to calculate the voltage received at the premises for the period of one year. The voltage drop to the $99.9^{\text {th }}$ percentile is recorded for each terminal section and for each phase. The simulation is performed twice, once for the previous design and once for the present design (Figure 5).

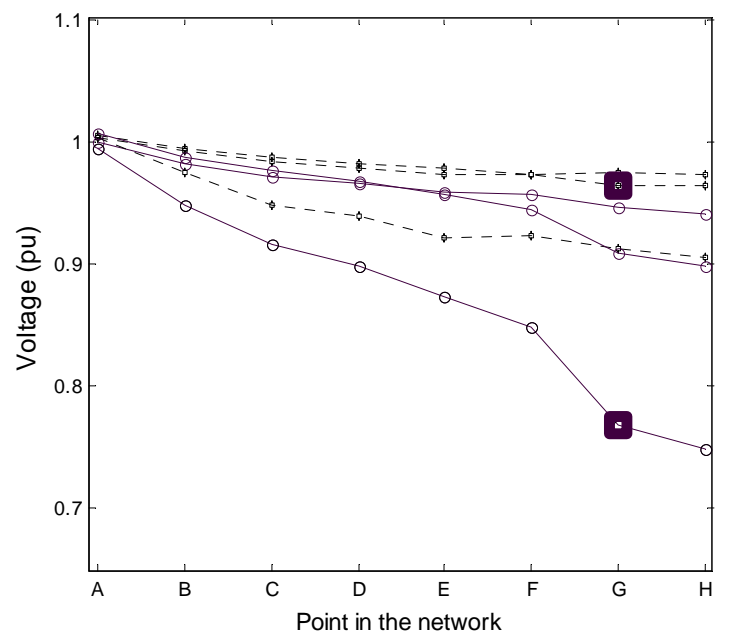

Fig. 5, Results of the Monte Carlo simulation for voltage drop. Legend is as for Figure 3.

The results agree very well with the PEP results. The voltage drop calculated using the Monte Carlo method is slightly less than that calculated using PEP but not outside agreement considering the equivalent confidence level.

Table 1. Voltages predicted for the case study premises using PEP and Monte Carlo simulatiopn for previous and present designs. The voltage for the Monte Carlo simulation is given at the $90^{\text {th }}, 99^{\text {th }}$ and $99.9^{\text {th }}$ percentiles.

\begin{tabular}{|l|c|c|c|c|}
\hline \multirow{2}{*}{ Design } & \multirow{2}{*}{ Traditional } & \multicolumn{3}{|c|}{ Monte Carlo simulation } \\
\cline { 3 - 5 } & & 90th & 99th & 99.9th \\
\hline \hline Present & 0.96 & 0.99 & 0.98 & 0.96 \\
\hline Previous & 0.73 & 0.94 & 0.89 & 0.84 \\
\hline
\end{tabular}

Numerical values for results are presented in Table 1. It is important to note that the voltage predicted at the $99^{\text {th }}$ percentile closely agrees with PEP and that the voltage at the $90^{\text {th }}$ percentile for the previous design was on the regulation limits.

To demonstrate the flexibility of the Monte Carlo method the expected yearly line losses for each conductor section in the previous and present design states is calculated (Table 2). Note that the electrical characteristics are kept as for the voltage drop simulation.

Table 2. Yearly conductor section losses in $\mathrm{kWh}$ per year, calculated using the Monte Carlo methodology.

\begin{tabular}{|l|c|c|}
\hline Section & Previous & Present \\
\hline \hline A & 3.6 & 3.3 \\
\hline B & 11.7 & 2.5 \\
\hline C & 8.9 & 1.8 \\
\hline D & 7.3 & 1.5 \\
\hline E & 6.4 & 1.3 \\
\hline F & 10.6 & 1.13 \\
\hline G & 9.7 & 0.9 \\
\hline H & 4.8 & 0.6 \\
\hline Total & 63 & 13.03 \\
\hline
\end{tabular}

\section{Phase Unbalance and Sampling Interval}

Phase balance is important to distribution networks; so that single phases do not become overloaded and so that loads that operate using all three phases (motors) do not experience adverse effects. Phase unbalance is not only the result of unequally placed loads within the network but also a natural consequence of diversity. The degree to which phase unbalance occurs within "balanced" networks can be examined using the Monte Carlo methodology.

We define an index to use as a measure of phase unbalance. This is taken to be the ratio of the neutral current to the sum of all phase currents (Equation 6).

$$
X=\frac{\left|I_{n}\right|}{\left|I_{R}\right|+\left|I_{Y}\right|+\left|I_{B}\right|}
$$




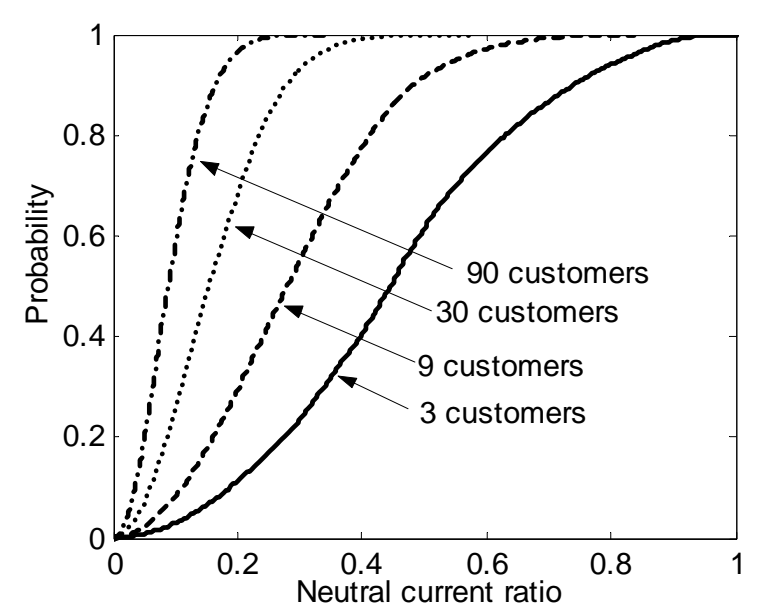

Fig. 6, Cumulative distribution functions formed for neutral current ratios for groups of $3,9,30$ and 90 customers with customers even balanced between phases.

The phase unbalance analysis (Figure 6) shows that the current in the neutral conductor when three customers are connected to the network is greater than the average current carried by any phase conductor for more than $70 \%$ of the time. Only once there are more than 9 customers connected to the network should it be considered that the neutral conductor could be downsized relative to the phase conductors (from the perspective of considering average load, not utilisation).

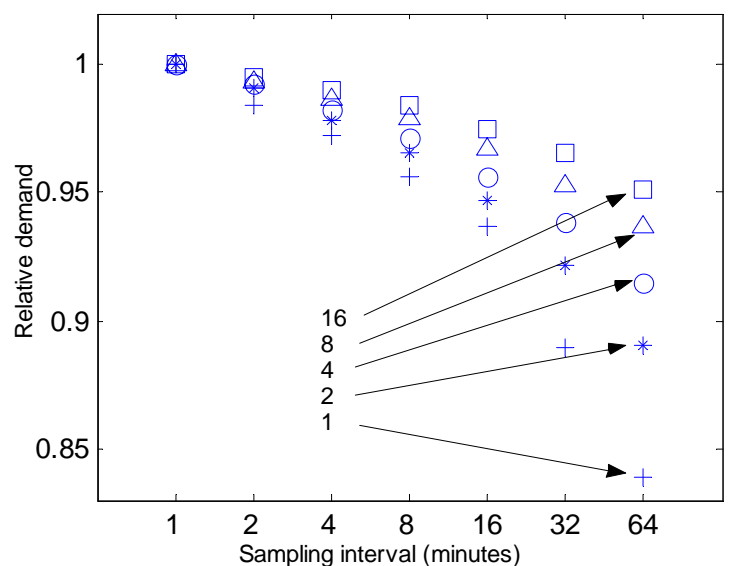

Fig. 7, Demand dependence upon sampling interval. The demand at the $99.9^{\text {th }}$ percentile relative to that measured using a 1-minute sampling interval is given for groups of 1,2, 4, 8, and 16 customers.

The dependence of demand on sampling interval was a point for investigation raised during the initial investigations of voltage drop methodology. The measured diversity is a direct result of the sampling interval and hence it is important to quantify when using a standard interval such as a $1 / 2$ hour. The analysis presented here bootstraps load profiles from the monitoring study dataset, and so is indicative not universal. It shows that a sampling interval of half-anhour may lead to an underestimation of demand at the highest confidence levels by up to $6 \%$.

\section{Conclusions}

A mathematical model for residential electricity demand based upon the premise that this process can be described as stochastic has been developed. This model has been shown to accurately simulate loading patterns observed within distribution networks and characterise diversity. The model has been deployed in a case study where Monte Carlo simulation was used to calculate the voltage drop. The voltages predicted by the Monte Carlo method have been compared with measured values and with the Prevalent Engineering Practice and found to be in good agreement. The flexibility of the Monte Carlo method has been demonstrated through the calculation of the line losses in the distribution network.

The Monte Carlo technique was further used to investigate phase unbalance. It was shown that phase unbalance is important for small groups of customers, and practices such as using a half-sized neutral conductor are not beneficial within distribution networks. The effect of sampling interval on predicted demand for group of customers has also been analysed. The important result is that for a group of 16 customers the demand at the $99^{\text {th }}$ percentile will be reduced by approximately $6 \%$ if a halfhour sampling interval is used.

Further work associated with this results presented in this paper includes:

- Using a more sophisticated model for the voltage drop calculation.

- Demonstrating the use of the Monte Carlo method for the effect of distributed generation within the LV network.

- Development of demand models for different customer types.

- Deploy the methodology within different electricity networks (particularly in networks where there are strong seasonal and temperature dependencies).

\section{Acknowledgements}

Many thanks to AuStral Engineering Associates, Delta Utilities Limited, Orion Limited, University of Otago, Industrial Research Limited. Thanks to Prof Pat Bodger, Electrical and Electronic Engineering, University of Canterbury.

\section{References}

[1] J Boggis, Diversity, Bias and Balance. Distribution of Electricity, pp 357-362, July 1953.

[2] IEEE Std 1159, IEEE recommended practice for monitoring power quality, 1995.

[3] H Wills, Spatial electric load forecasting. Marcel Dekker, New York, 1996.

[4] T Eichelbaum, Electricity Regulations, Government of New Zealand, 1997.

[5] BS EN 50 160, Voltage Characteristics of electricity supplied by public distribution systems. British Standards Institution, 2000

[6] C Warren, C Burns, Home Power Quality - the Niagara Mohawk study. In Proc. 1994 IEEE power engineering society; transmission, and distribution conference, pp 634-638, 1994. 
[7] P. Hyland, D. McQueen, Using a Residential Electricity Model for Sizing Low Voltage Networks. In Proc. Electricity Engineer's Association of New Zealand conference 2000.

[8] R.Herman, J.Kritzinger, General probabilistic voltage drop calculation method for LV distribution networks based on a beta p.d.f. load model. Electric power systems research, Vol. 46, pp 45-49, 1998.

[9]A Capasso, W Grattieri, R Lamedica, A Prudenzi, A bottomup approach to residential load modelling. IEEE transactions on power systems, 9 No 2, pp 957-963, May 1994.

[10] D. McQueen, Mathematical Modelling of Residential Electricity Demand and Engineering Applications. Thesis submitted for the Degree of Masters of Applied Science, University of Otago, New Zealand, 2002.

[11] D. McQueen, P. Hyland, Simulation of Distribution Trransformer Loading. In Proc. $37^{\text {th }}$ International Universities Power Engineering Conference 2002.

[12] Dunedin Electricity Limited, System Impedance Manual quality reference document, 1995.

\section{Appendix A}

Network impedance and conductor characteristics for the voltage drop case study.

Table 3. Parameters of conductor sections.

\begin{tabular}{|c|c|c|c|c|c|}
\hline \multirow{2}{*}{ Section } & \multirow{2}{*}{ Length $(\mathrm{m})$} & \multicolumn{4}{|c|}{ Conductor } \\
\cline { 3 - 6 } & & \multicolumn{2}{|c|}{ Previous design } & \multicolumn{2}{|c|}{ Present design } \\
\cline { 3 - 6 } & & Phase & Neutral & Phase & Neutral \\
\hline A & 23 & $37 ; 0.083$ & $19 ; 0.083$ & $37 ; 0.083$ & $19 ; 0.083$ \\
B & 51 & $19 ; 0.052$ & $19 ; 0.132 \mathrm{Al}$ & $19 ; 0.132 \mathrm{Al}$ & $37 ; 0.072$ \\
C & 20 & $19 ; 0.052$ & $19 ; 0.132 \mathrm{Al}$ & $19 ; 0.132 \mathrm{Al}$ & $37 ; 0.072$ \\
D & 35 & $19 ; 0.052$ & $19 ; 0.132 \mathrm{Al}$ & $19 ; 0.132 \mathrm{Al}$ & $37 ; 0.072$ \\
E & 21 & $19 ; 0.052$ & $19 ; 0.132 \mathrm{Al}$ & $19 ; 0.132 \mathrm{Al}$ & $37 ; 0.072$ \\
F & 37 & $7 ;$ No 18 & No 10 & $7 ; 0.186 \mathrm{Al}$ & $19 ; 0.083$ \\
G & 42 & $7 ;$ No 18 & No 10 & $7 ; 0.186 \mathrm{Al}$ & $19 ; 0.083$ \\
H & 42 & $7 ;$ No 18 & No 10 & $7 ; 0.186 \mathrm{Al}$ & $19 ; 0.083$ \\
\hline
\end{tabular}

Table 4. Parameters of conductor types.

\begin{tabular}{|l|l|c|c|}
\hline Type & Description & $\begin{array}{l}\text { Resistance } \\
\text { (ohms/km) }\end{array}$ & GMR (mm) \\
\hline \hline $7 ;$ No 18 & $\begin{array}{l}7 \text { strands of Number } \\
18 \text { wire } \\
\text { Number 10 wire } 10\end{array}$ & 2.11 & 0.27 \\
$19 ; 0.052$ & $\begin{array}{l}\text { 19 strands of 0.052" } \\
\text { copper wire }\end{array}$ & 0.69 & 1.63 \\
$19 ; 0.083$ & $\begin{array}{l}19 \text { strands of 0.083" } \\
\text { copper wire } \\
37 ; 0.072\end{array}$ & 0.27 & 3.98 \\
$7 ; 0.186 \mathrm{Al}$ strands of 0.083" & $\begin{array}{l}\text { lopper wire } \\
7 \text { strands of 0.186" } \\
\text { aluminium wire } \\
19 \text { strands of 0.132" } \\
\text { aluminium wire }\end{array}$ & 0.14 & 5.68 \\
\hline
\end{tabular}

